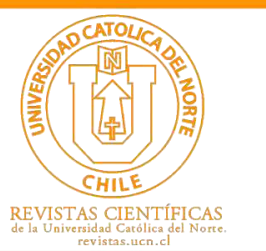

\title{
Study of topology of block shift networks via topological indices
}

\author{
Murat Cancan ${ }^{1}$ (0) orcid.org/0000-0002-8606-2274. \\ Iftikhar Ahmad'º orcid.org/0000-0001-5879-5801 \\ Sarfarz Ahmad ${ }^{3}$
}

${ }^{1}$ Van Yznc Yil University, Faculty of Education, Van, Turkey.

m m cencen@yahoo.com

${ }^{2}$ COMSATS University Islamabad, Dept. of Mathematics, Lahore, Pakistan.

iffi6301@gmail.com

${ }^{3}$ COMSATS University Islamabad, Dept. of Mathematics, Lahore, Pakistan.

iffi6301@gmail.com

Received: February 2020 | Accepted: May 2020

\begin{abstract}
:
Topological indices(TIs) are important numerical number associate with the molecular graph of a chemical structure/compound because due to these parameters, one can guess almost all properties of concerned structure/compound with our performing experiments. In recent years, huge amount work has been done for calculating degreedependent indices for different structures/compouds. In order to compute TIs, one need to do many calculations. Our aim of this paper is to present a simple method to compute degree-dependent TIs. We computed M-polynomials for Block Shift Networks and with the help of this simple algebraic polynomials, we recovered nine important TIs for Block Shift Networks. Our work is important for chemists, physicians and pharmaceutical industry.
\end{abstract}

Keywords: Topological index; Physical properties of compounds; Graph; Network.

MSC (2020): 05C12, 05C90, 05C78.

Cite this article as (IEEE citation style):

M. Cancan, I. Ahmad, and S. Ahmad, "Study of topology of block shift networks via topological indices", Proyecciones (Antofagasta, On line), vol. 39, no. 4, pp. 887-902, Aug. 2020, doi: 10.22199/issn.0717-6279-2020-04-0055.

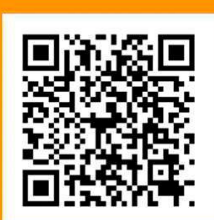

Article copyright: (C) 2020 Murat Cancan, Iftikhar Ahmad, and Sarfarz Ahmad. This is an open access article distributed under the terms of the Creative Commons License, which permits unrestricted use and distribution provided the original author and source are credited. 


\section{Introduction}

The representation of a chemical structure with the help of TIs is an interesting topic in chemistry and boi-informatics [8]. This approach help us in deciding properties of chemical compounds without performing experiments [8]. TIs in this way an important tool for chemists and mathematician are interesting in computing TIs of different chemical structures [1]. The first topological index was Wiener index defined in 1947 [29]. After the success of this index [13,30], Randic index (RI) was defined in [24]. The formula for RI is:

$$
R_{-\frac{1}{2}}=\sum_{u v \in E(G)} \frac{1}{\sqrt{d_{u} d_{v}}} .
$$

This is the most successful index till now and has been studied extensively $[24,25]$. Zagreb indices (ZIs) are also oldest TIs and the first ZI and second $\mathrm{ZI}$ are defined in $[12]$ as:

$$
M_{1}(G)=\sum_{u v \in E(G)} d_{u}+d_{v}
$$

and

$$
M_{2}(G)=\sum_{u v \in E(G)} d_{u} d_{v}
$$

For details about applications of these indices, we refer $[4,16,23,25,27,28]$.

The formula for the redefined ZI [18] is:

$$
{ }^{m} M_{2}(G)=\sum_{u v \in E(G)} \frac{1}{d_{u} d_{v}} .
$$

The mathematical formula for the symmetric division index (SDI) [11] is:

$$
S D I(G)=\sum_{u v \in E(G)}\left\{\frac{\min \left\{d_{u}, d_{v}\right\}}{\max \left\{d_{u}, d_{v}\right\}}+\frac{\max \left\{d_{u}, d_{v}\right\}}{\min \left\{d_{u}, d_{v}\right\}}\right\}
$$

Other interesting TIs is harmonic index (HI) [6] which is a variant of RI and mathematical formula for this TI is:

$$
H(G)=\sum_{u v \in E(G)} \frac{2}{d_{u} d_{v}} .
$$


The other two TIs of our interest are inverse sum index (ISI) [2] and Augmented ZI [7] and these TIs are defined as:

$$
I(G)=\sum_{u v \in E(G)} \frac{d_{u} d_{v}}{d_{u}+d_{v}}
$$

and

$$
A(G)=\sum_{u v \in E(G)}\left\{\frac{d_{u} d_{v}}{d_{u}+d_{v}-2}\right\}^{3} .
$$

The above defined TIs are important for the researcher working in chemistry [3.5.14.15.17.22.23.26] and huge computational work is required for calculating these indices $[10,22]$. In order to reduce computational work [30], M-polynomial has been introduced and one can get almost every degreedependent index from this simple polynomial $[20,27]$. The mathematical formula of this polynomial is

$$
M(G ; x, y)=\sum_{u v \in E(G)}\left(x^{d_{u}} y^{d_{v}}\right) .
$$

The relation of this polynomial with above mentioned nine TIs can be found in $[21,22]$.

In this paper, our aim is to compute M-polynomial for Block-Shift Network.

The Block shift network $B S N-1$ is the shuffle-exchange network ndimensional hypercube, while block shift network $B S N-2$ is the complete network, as shown in Figure 1.1 and Figure 1.2 respectively. Let G be a block shift network. It can be seen from Figure 1 that the number of vertices and edges in $(B S N-1)$ are $16 a^{2}$ and $24 a^{2}-2$ respectively. From Figure 2 , one can observe that the number of vertices and edges in $(B S N-2)$ are $16 a^{2}$ and $32 a^{2}-2$ respectively. 


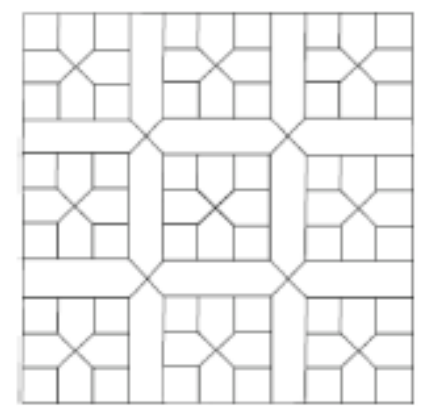

Figure 1.1: $(B S N-1)_{(3 \times 3)}$

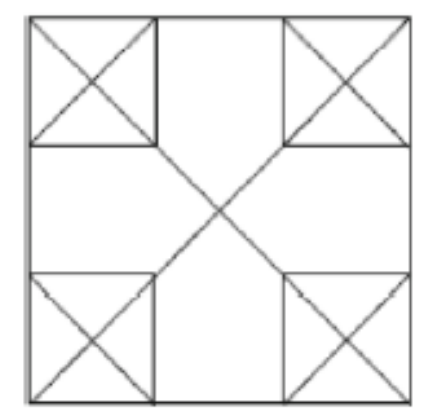

Figure 1.2: $(B S N-2)_{(1 \times 1)}$

\section{Main Results}

In this section, we compute M-polynomials of understudy networks and recover nine TIs from these polynomials.

2.1. Results for $(B S N-1)_{(n \times n)}$

Theorem 2.1. Let $(B S N-1)_{(n \times n)}$ be the block shift Network, then

$$
M(G ; x, y)=8 x^{2} y^{3}+\left(24 n^{2}-10\right) x^{3} y^{3} .
$$


Proof. The $(B S N-1)_{(n \times n)}$ network has following two type of edges based on the degree of end vertices:

$E_{\{2,3\}}\left((B S N-1)_{(n \times n)}\right)=\left\{u v \in E\left((B S N-1)_{(n \times n)}\right): d_{u}=2, d_{v}=3\right\}$,

$E_{\{3,3\}}\left((B S N-1)_{(n \times n)}\right)=\left\{u v \in E\left((B S N-1)_{(n \times n)}\right): d_{u}=3, d_{v}=3\right\}$,

such that

$\left|E_{\{2,3\}}\left((B S N-1)_{(n \times n)}\right)\right|=8$,

$\left|E_{\{3,3\}}\left((B S N-1)_{(n \times n)}\right)\right|=\left(24 n^{2}-10\right)$,

Now from the definition of M-polynomial, we have

$$
\begin{aligned}
M(G ; x, y) & =\sum_{\delta \leq i \leq j \leq \Delta} m_{i j} x^{i} y^{j} \\
& =\sum_{2 \leq 3} m_{23} x^{2} y^{3}+\sum_{3 \leq 3} m_{33} x^{3} y^{3} \\
& =\left|E_{\{2,3\}}\left((B S N-1)_{(n \times n)}\right)\right| x^{2} y^{3}+\left|E_{\{3,3\}}\left((B S N-1)_{(n \times n)}\right)\right| x^{3} y^{3} \\
& =8 x^{2} y^{3}+\left(24 n^{2}-10\right) x^{3} y^{3}
\end{aligned}
$$

Corollary 2.2. Let $(B S N-1)_{(n \times n)}$ be the block shift Network, then

$$
M_{1}\left((B S N-1)_{(n \times n)}\right)=144 n^{2}-20
$$

Proof. Let $f(x, y)=M(G ; x, y)=8 x^{2} y^{3}+\left(24 n^{2}-10\right) x^{3} y^{3}$.

Then

$D_{x}(f(x, y))=16 x^{2} y^{3}+3\left(24 n^{2}-10\right) x^{3} y^{3}$,

$D_{y}(f(x, y))=24 x^{2} y^{3}+3\left(24 n^{2}-10\right) x^{3} y^{3}$.

Hence

$$
\begin{aligned}
M_{1}\left((B S N-1)_{(n \times n)}\right) & =D_{x} f+\left.D_{y} f\right|_{x=y=1} \\
& =144 n^{2}-20 .
\end{aligned}
$$

Corollary 2.3. Let $(B S N-1)_{(n \times n)}$ be the block shift Network, then

$$
M_{2}\left((B S N-1)_{(n \times n)}\right)=216 n^{2}-42
$$


Proof. Let $f(x, y)=M(G ; x, y)=8 x^{2} y^{3}+\left(24 n^{2}-10\right) x^{3} y^{3}$.

Then

$D_{y}(f(x, y))=24 x^{2} y^{3}+3\left(24 n^{2}-10\right) x^{3} y^{3}$,

$D_{x} D_{y}(f(x, y))=48 x^{2} y^{3}+9\left(24 n^{2}-10\right) x^{3} y^{3}$,

Hence

$$
\begin{aligned}
M_{2}\left((B S N-1)_{(n \times n)}\right) & =\left.D_{x} D_{y} f\right|_{x=y=1} \\
& =216 n^{2}-42 .
\end{aligned}
$$

Corollary 2.4. Let $(B S N-1)_{(n \times n)}$ be the block shift Network, then

$$
{ }^{m} M_{2}\left((B S N-1)_{(n \times n)}\right)=\frac{8 n^{2}}{3}+\frac{2}{9}
$$

Proof. Let $f(x, y)=M(G ; x, y)=8 x^{2} y^{3}+\left(24 n^{2}-10\right) x^{3} y^{3}$.

Then

$S_{y}(f(x, y))=\frac{8 x^{2} y^{3}}{3}+\frac{\left(24 n^{2}-10\right) x^{3} y^{3}}{3}$,

$S_{x} S_{y}(f(x, y))=\frac{4 x^{2} y^{3}}{3}+\frac{\left(24 n^{2}-10\right) x^{3} y^{3}}{9}$.

Hence

$$
\begin{aligned}
{ }^{m} M_{2}\left((B S N-1)_{(n \times n)}\right) & =\left.S_{x} S_{y} f\right|_{x=y=1} \\
& =\frac{8 n^{2}}{3}+\frac{2}{9} .
\end{aligned}
$$

Corollary 2.5. Let $(B S N-1)_{(n \times n)}$ be the block shift Network, then

$$
R_{\alpha}\left(\left((B S N-1)_{(n \times n)}\right)\right)=24^{\alpha} 2^{\alpha}+3^{2 \alpha}\left(24 n^{2}-10\right)
$$

Proof. Let $f(x, y)=M(G ; x, y)=8 x^{2} y^{3}+\left(24 n^{2}-10\right) x^{3} y^{3}$.

Then

$D_{y}^{\alpha}(f(x, y))=24^{\alpha} x^{2} y^{3}+3^{\alpha}\left(24 n^{2}-10\right) x^{3} y^{3}$.

$D_{x}^{\alpha} D_{y}^{\alpha}\left((f(x, y))=24^{\alpha} 2^{\alpha} x^{2} y^{3}+3^{2 \alpha}\left(24 n^{2}-10\right) x^{3} y^{3}\right.$.

Hence

$$
\begin{aligned}
R_{\alpha}\left(\left((B S N-1)_{(n \times n)}\right)\right)= & =\left.D_{x}^{\alpha} D_{y}^{\alpha} f\right|_{x=y=1} \\
& =24^{\alpha} 2^{\alpha}+3^{2 \alpha}\left(24 n^{2}-10\right) .
\end{aligned}
$$


Corollary 2.6. Let $(B S N-1)_{(n \times n)}$ be the block shift Network, then

$$
R R_{\alpha}\left(\left((B S N-1)_{(n \times n)}\right)\right)=\frac{8^{\alpha}}{3^{\alpha} 2^{\alpha}}+\frac{24 n^{2}-10}{3^{2 \alpha}}
$$

Proof. Let $f(x, y)=M(G ; x, y)=8 x^{2} y^{3}+\left(24 n^{2}-10\right) x^{3} y^{3}$.

Then

$S_{y}^{\alpha}(f(x, y))=\frac{8^{\alpha} x^{2} y^{3}}{3^{\alpha}}+\frac{\left(24 n^{2}-10\right) x^{3} y^{3}}{3^{\alpha}}$.

$S_{x}^{\alpha} S_{y}^{\alpha}\left((f(x, y))=\frac{8^{\alpha} x^{2} y^{3}}{3^{\alpha} 2^{\alpha}}+\frac{\left(24 n^{2}-10\right) x^{3} y^{3}}{3^{\alpha} 3^{\alpha}}\right.$.

Hence

$$
\begin{aligned}
R R_{\alpha}\left(\left((B S N-1)_{(n \times n)}\right)\right)= & =\left.S_{x}^{\alpha} S_{y}^{\alpha} f\right|_{x=y=1} \\
& =\frac{8^{\alpha}}{3^{\alpha} 2^{\alpha}}+\frac{\left(24 n^{2}-10\right.}{3^{2 \alpha}} .
\end{aligned}
$$

Corollary 2.7. Let $(B S N-1)_{(n \times n)}$ be the block shift Network, then

$$
S S D\left((B S N-1)_{(n \times n)}\right)=\frac{52}{3}+2\left(24 n^{2}-10\right)
$$

Proof. Let $f(x, y)=M(G ; x, y)=8 x^{2} y^{3}+\left(24 n^{2}-10\right) x^{3} y^{3}$.

Then

$S_{x} D_{y}(f(x, y))=12 x^{2} y^{3}+\left(24 n^{2}-10\right) x^{3} y^{3}$,

$D_{x} S_{y}(f(x, y))=\frac{16 x^{2} y^{3}}{3}+\left(24 n^{2}-10\right) x^{3} y^{3}$.

Hence

$$
\begin{aligned}
S S D\left((B S N-1)_{(n \times n)}\right) & =\left.\left(D_{x} S_{y} f+S_{x} D_{y} f\right)\right|_{x=y=1} \\
& =\frac{52}{3}+2\left(24 n^{2}-10\right) .
\end{aligned}
$$

Corollary 2.8. Let $(B S N-1)_{(n \times n)}$ be the block shift Network, then

$$
H\left((B S N-1)_{(n \times n)}\right)=2\left(\frac{8}{5}+\frac{\left(24 n^{2}-10\right)}{6}\right)
$$


Proof. Let $f(x, y)=M(G ; x, y)=8 x^{2} y^{3}+\left(24 n^{2}-10\right) x^{3} y^{3}$.

Then

$J(f(x, y))=8 x^{5}+\left(24 n^{2}-10\right) x^{6}$,

$2 S_{x} J(f(x, y))=2\left(\frac{8 x^{5}}{5}+\frac{\left(24 n^{2}-10\right) x^{6}}{6}\right)$.

Hence

$$
\begin{aligned}
H\left((B S N-1)_{(n \times n)}\right) & =\left.2 S_{x} J f\right|_{x=1} \\
& =2\left(\frac{8}{5}+\frac{\left(24 n^{2}-10\right)}{6}\right) .
\end{aligned}
$$

Corollary 2.9. Let $(B S N-1)_{(n \times n)}$ be the block shift Network, then

$$
I\left((B S N-1)_{(n \times n)}\right)=\frac{48}{5}+\frac{3\left(24 n^{2}-10\right)}{2}
$$

Proof. Let $f(x, y)=M(G ; x, y)=8 x^{2} y^{3}+\left(24 n^{2}-10\right) x^{3} y^{3}$.

Then

$D_{y}(f(x, y))=24 x^{2} y^{3}+3\left(24 n^{2}-10\right) x^{3} y^{3}$,

$D_{x} D_{y}(f(x, y))=48 x^{2} y^{3}+9\left(24 n^{2}-10\right) x^{3} y^{3}$,

$J D_{x} D_{y}(f(x, y))=48 x^{5}+9\left(24 n^{2}-10\right) x^{6}$,

$S_{x} J D_{x} D_{y}(f(x, y))=\frac{48 x^{5}}{5}+\frac{9 x^{6}\left(24 n^{2}-10\right)}{6}$.

Hence

$$
\begin{aligned}
I\left((B S N-1)_{(n \times n)}\right) & =\left.S_{x} J D_{x} D_{y} f\right|_{x=1} \\
& =\frac{48}{5}+\frac{3\left(24 n^{2}-10\right)}{2} .
\end{aligned}
$$

Corollary 2.10. Let $(B S N-1)_{(n \times n)}$ be the block shift Network, then

$$
A\left((B S N-1)_{(n \times n)}\right)=\frac{2187 n^{2}}{8}-\frac{1597}{32}
$$

Proof. Let $f(x, y)=M(G ; x, y)=8 x^{2} y^{3}+\left(24 n^{2}-10\right) x^{3} y^{3}$.

Then

$D_{y}^{3}(f(x, y))=216 x^{2} y^{3}+27\left(24 n^{2}-10\right) x^{3} y^{3}$, 
$D_{x}^{3} D_{y}^{3}(f(x, y))=1728 x^{2} y^{3}+729\left(24 n^{2}-10\right) x^{3} y^{3}$,

$J D_{x}^{3} D_{y}^{3}(f(x, y))=1728 x^{5}+729\left(24 n^{2}-10\right) x^{6}$,

$Q_{-2} J D_{x}^{3} D_{y}^{3}(f(x, y))=1728 x^{3}+729\left(24 n^{2}-10\right) x^{4}$,

$S_{x}^{3} Q_{-2} J D_{x}^{3} D_{y}^{3}(f(x, y))=64 x^{2}+\frac{729 x^{4}\left(24 n^{2}-10\right)}{64}$.

Hence

$$
\begin{aligned}
A\left((B S N-1)_{(n \times n)}\right) & =\left.S_{x}^{3} Q_{-2} J D_{x}^{3} D_{y}^{3} f\right|_{x=1} \\
& =\frac{2187 n^{2}}{8}-\frac{1597}{32}
\end{aligned}
$$

2.2. Results for $(B S N-2)_{(n \times n)}$

Theorem 2.11. Let $(B S N-2)_{(n \times n)}$ be the block shift Network, then

$$
M(G ; x, y)=12 x^{3} y^{4}+\left(32 n^{2}-14\right) x^{4} y^{4} .
$$

Proof. The $(B S N-2)_{(n \times n)}$ network has following two type of edges based on the degree of end vertices:

$E_{\{3,4\}}\left((B S N-2)_{(n \times n)}\right)=\left\{u v \in E\left((B S N-2)_{(n \times n)}\right): d_{u}=3, d_{v}=4\right\}$,

$E_{\{4,4\}}\left((B S N-2)_{(n \times n)}\right)=\left\{u v \in E\left((B S N-2)_{(n \times n)}\right): d_{u}=4, d_{v}=4\right\}$,

such that

$\mid \begin{aligned} & E_{\{3,4\}}\left((B S N-2)_{(n \times n)}\right) \mid=12, \\ & E_{\{4,4\}}\left((B S N-2)_{(n \times n)}\right) \mid=\left(32 n^{2}-14\right),\end{aligned}$

Now from the definition of M-polynomial, we have

$$
\begin{aligned}
M(G ; x, y) & =\sum_{\delta \leq i \leq j \leq \Delta} m_{i j} x^{i} y^{j} \\
& =\sum_{3 \leq 4} m_{34} x^{3} y^{4}+\sum_{4 \leq 4} m_{44} x^{4} y^{4} \\
& =\left|E_{\{3,4\}}\left((B S N-2)_{(n \times n)}\right)\right| x^{3} y^{4}+\left|E_{\{4,4\}}\left((B S N-2)_{(n \times n)}\right)\right| x^{4} y^{4} \\
& =12 x^{3} y^{4}+\left(32 n^{2}-14\right) x^{4} y^{4} .
\end{aligned}
$$

Corollary 2.12. Let $(B S N-2)_{(n \times n)}$ be the block shift Network, then

$$
M_{1}\left((B S N-2)_{(n \times n)}\right)=256 n^{2}-28
$$


Proof. Let $f(x, y)=M(G ; x, y)=12 x^{3} y^{4}+\left(32 n^{2}-14\right) x^{4} y^{4}$.

Then

$D_{x}(f(x, y))=36 x^{3} y^{4}+4\left(32 n^{2}-14\right) x^{4} y^{4}$,

$D_{y}(f(x, y))=48 x^{3} y^{4}+4\left(32 n^{2}-14\right) x^{4} y^{4}$.

Hence

$$
\begin{aligned}
M_{1}\left((B S N-2)_{(n \times n)}\right) & =D_{x} f+\left.D_{y} f\right|_{x=y=1} \\
& =256 n^{2}-28 .
\end{aligned}
$$

Corollary 2.13. Let $(B S N-2)_{(n \times n)}$ be the block shift Network, then

$$
M_{2}\left((B S N-2)_{(n \times n)}\right)=512 n^{2}-80
$$

Proof. Let $f(x, y)=M(G ; x, y)=12 x^{3} y^{4}+\left(32 n^{2}-14\right) x^{4} y^{4}$.

Then

$D_{y}(f(x, y))=48 x^{3} y^{4}+4\left(32 n^{2}-14\right) x^{4} y^{4}$,

$D_{x} D_{y}(f(x, y))=144 x^{3} y^{4}+16\left(32 n^{2}-14\right) x^{4} y^{4}$,

Hence

$$
\begin{aligned}
M_{2}\left((B S N-2)_{(n \times n)}\right) & =\left.D_{x} D_{y} f\right|_{x=y=1} \\
& =512 n^{2}-80 .
\end{aligned}
$$

Corollary 2.14. Let $(B S N-2)_{(n \times n)}$ be the block shift Network, then

$$
{ }^{m} M_{2}\left((B S N-2)_{(n \times n)}\right)=2 n^{2}+\frac{1}{8}
$$

Proof. Let $f(x, y)=M(G ; x, y)=12 x^{3} y^{4}+\left(32 n^{2}-14\right) x^{4} y^{4}$.

Then

$S_{y}(f(x, y))=3 x^{3} y^{4}+\frac{\left(32 n^{2}-14\right) x^{4} y^{4}}{4}$,

$S_{x} S_{y}(f(x, y))=x^{3} y^{4}+\frac{\left(32 n^{2}-14\right) x^{4} y^{4}}{16}$.

Hence

$$
\begin{aligned}
{ }^{m} M_{2}\left((B S N-2)_{(n \times n)}\right) & =\left.S_{x} S_{y} f\right|_{x=y=1} \\
& =2 n^{2}+\frac{1}{8}
\end{aligned}
$$


Corollary 2.15. Let $(B S N-2)_{(n \times n)}$ be the block shift Network, then

$$
R_{\alpha}\left(\left((B S N-2)_{(n \times n)}\right)\right)=48^{\alpha} 3^{\alpha}+4^{2 \alpha}\left(32 n^{2}-14\right)
$$

Proof. Let $f(x, y)=M(G ; x, y)=12 x^{3} y^{4}+\left(32 n^{2}-14\right) x^{4} y^{4}$.

Then

$D_{y}^{\alpha}(f(x, y))=48^{\alpha} x^{3} y^{4}+4^{\alpha}\left(32 n^{2}-14\right) x^{4} y^{4}$.

$D_{x}^{\alpha} D_{y}^{\alpha}\left((f(x, y))=48^{\alpha} 3^{\alpha} x^{3} y^{4}+4^{2 \alpha}\left(32 n^{2}-14\right) x^{4} y^{4}\right.$.

Hence

$$
\begin{aligned}
R_{\alpha}\left(\left((B S N-2)_{(n \times n)}\right)\right)= & =\left.D_{x}^{\alpha} D_{y}^{\alpha} f\right|_{x=y=1} \\
& =48^{\alpha} 3^{\alpha}+4^{2 \alpha}\left(32 n^{2}-14\right) .
\end{aligned}
$$

Corollary 2.16. Let $(B S N-2)_{(n \times n)}$ be the block shift Network, then

$$
R R_{\alpha}\left(\left((B S N-2)_{(n \times n)}\right)\right)=1+\frac{32 n^{2}-14}{4^{2 \alpha}} .
$$

Proof. Let $f(x, y)=M(G ; x, y)=12 x^{3} y^{4}+\left(32 n^{2}-14\right) x^{4} y^{4}$.

Then

$S_{y}^{\alpha}(f(x, y))=3^{\alpha} x^{3} y^{4}+\frac{\left(32 n^{2}-14\right) x^{4} y^{4}}{4^{\alpha}}$

$S_{x}^{\alpha} S_{y}^{\alpha}\left((f(x, y))=x^{3} y^{4}+\frac{\left(32 n^{2}-14\right) x^{4} y^{4}}{4^{2 \alpha}}\right.$.

Hence

$$
\begin{aligned}
R R_{\alpha}\left(\left((B S N-2)_{(n \times n)}\right)\right)= & =\left.S_{x}^{\alpha} S_{y}^{\alpha} f\right|_{x=y=1} \\
& =1+\frac{32 n^{2}-14}{4^{2 \alpha}} .
\end{aligned}
$$

Corollary 2.17. Let $(B S N-2)_{(n \times n)}$ be the block shift Network, then

$$
S S D\left((B S N-2)_{(n \times n)}\right)=64 n^{2}-3
$$


Proof. Let $f(x, y)=M(G ; x, y)=12 x^{3} y^{4}+\left(32 n^{2}-14\right) x^{4} y^{4}$.

Then

$S_{x} D_{y}(f(x, y))=16 x^{3} y^{4}+\left(32 n^{2}-14\right) x^{4} y^{4}$, $D_{x} S_{y}(f(x, y))=9 x^{3} y^{4}+\left(32 n^{2}-14\right) x^{4} y^{4}$.

Hence

$$
\begin{aligned}
S S D\left((B S N-2)_{(n \times n)}\right) & =\left.\left(D_{x} S_{y} f+S_{x} D_{y} f\right)\right|_{x=y=1} \\
& =64 n^{2}-3 .
\end{aligned}
$$

Corollary 2.18. Let $(B S N-2)_{(n \times n)}$ be the block shift Network, then

$$
H\left((B S N-2)_{(n \times n)}\right)=2\left(\frac{12}{7}+\frac{\left(32 n^{2}-14\right)}{8}\right)
$$

Proof. Let $f(x, y)=M(G ; x, y)=12 x^{3} y^{4}+\left(32 n^{2}-14\right) x^{4} y^{4}$.

Then

$J(f(x, y))=12 x^{7}+\left(32 n^{2}-14\right) x^{8}$,

$2 S_{x} J(f(x, y))=2\left(\frac{12 x^{7}}{7}+\frac{\left(32 n^{2}-14\right) x^{8}}{8}\right)$.

Hence

$$
\begin{aligned}
H\left((B S N-2)_{(n \times n)}\right) & =\left.2 S_{x} J f\right|_{x=1} \\
& =2\left(\frac{12}{7}+\frac{\left(32 n^{2}-14\right)}{8}\right) .
\end{aligned}
$$

Corollary 2.19. Let $(B S N-2)_{(n \times n)}$ be the block shift Network, then

$$
I\left((B S N-2)_{(n \times n)}\right)=64 n^{2}-\frac{52}{7}
$$

Proof. Let $f(x, y)=M(G ; x, y)=12 x^{3} y^{4}+\left(32 n^{2}-14\right) x^{4} y^{4}$.

Then

$D_{y}(f(x, y))=48 x^{3} y^{4}+4\left(32 n^{2}-14\right) x^{4} y^{4}$,

$D_{x} D_{y}(f(x, y))=144 x^{3} y^{4}+16\left(32 n^{2}-14\right) x^{4} y^{4}$,

$J D_{x} D_{y}(f(x, y))=144 x^{7}+16\left(32 n^{2}-14\right) x^{8}$, 
$S_{x} J D_{x} D_{y}(f(x, y))=\frac{144 x^{7}}{7}+2 x^{8}\left(32 n^{2}-14\right)$.

Hence

$$
\begin{aligned}
I\left((B S N-2)_{(n \times n)}\right) & =\left.S_{x} J D_{x} D_{y} f\right|_{x=1} \\
& =64 n^{2}-\frac{52}{7} .
\end{aligned}
$$

Corollary 2.20. Let $(B S N-2)_{(n \times n)}$ be the block shift Network, then

$$
A\left((B S N-2)_{(n \times n)}\right)=\frac{16384 n^{2}}{27}-\frac{336128}{3375}
$$

Proof. Let $f(x, y)=M(G ; x, y)=12 x^{3} y^{4}+\left(32 n^{2}-14\right) x^{4} y^{4}$.

Then

$D_{y}^{3}(f(x, y))=768 x^{3} y^{4}+64\left(32 n^{2}-14\right) x^{4} y^{4}$,

$D_{x}^{3} D_{y}^{3}(f(x, y))=20736 x^{3} y^{4}+4096\left(32 n^{2}-14\right) x^{4} y^{4}$,

$J D_{x}^{3} D_{y}^{3}(f(x, y))=20736 x^{7}+4096\left(32 n^{2}-14\right) x^{8}$,

$Q_{-2} J D_{x}^{3} D_{y}^{3}(f(x, y))=20736 x^{5}+4096\left(32 n^{2}-14\right) x^{6}$,

$S_{x}^{3} Q_{-2} J D_{x}^{3} D_{y}^{3}(f(x, y))=\frac{20736 x^{5}}{125}+\frac{512 x^{6}\left(32 n^{2}-14\right)}{27}$.

Hence

$$
\begin{aligned}
A\left((B S N-2)_{(n \times n)}\right) & =\left.S_{x}^{3} Q_{-2} J D_{x}^{3} D_{y}^{3} f\right|_{x=1} \\
& =\frac{16384 n^{2}}{27}-\frac{336128}{3375} .
\end{aligned}
$$

\section{Conclusion}

In this paper, we computed several degree-dependent indices for Block Shift Networks. We used edge division for this purpose and with the help of our results, one can guess the properties for Block Shift Networks.

\section{Acknowledgment}

This paper is supported by University natural science research project in Anhui province. Item no: kJ2017A622. 


\section{References}

[1] M. S. Anjum and M. U. Safdar, "K Banhatti and K hyper-Banhatti indices of nanotubes", Engineering and applied science letters, vol. 2, no. 1, pp. 19-37, Mar. 2019, doi: 10.30538/psrp-easl2019.0013

[2] A. T. Balaban, "Highly discriminating distance-based topological index", Chemical physics letters, vol. 89, no. 5, pp. 399-404, Jul. 1982, doi: 10.1016/0009-2614(82)80009-2

[3] G. Caporossi, I. Gutman, P. Hansen, and L. Pavlović, "Graphs with maximum connectivity index", Computational biology and chemistry, vol. 27, no. 1, pp. 85-90, Feb. 2003, doi: 10.1016/S0097-8485(02)00016-5

[4] N. De, "Hyper Zagreb index of bridge and Chain graphs", Open journal of mathematical sciences, pp. 1-17, 2018, doi: 10.30538/oms2018.0013

[5] E. Deutsch and Klavžar, "S. M-Polynomial, and degree-based topological indices", Iranian journal of mathematical chemistry, vol. 6, no. 2, pp. 93-102, 2015, doi: 10.22052/IJMC.2015.10106

[6] O. Favaron, M. Mahéo, and J.-F. Saclé, "Some eigenvalue properties in graphs (conjectures of Graffiti - II)", Discrete mathematics, vol. 111, no. 1-3, pp. 197-220, Feb. 1993, doi: 10.1016/0012-365X(93)90156-N

[7] B. Furtula, A. Graovac, and D. Vukičević, "Augmented Zagreb index", Journal of mathematical chemistry, vol. 48, no. 2, pp. 370-380, Apr. 2010, doi: 10.1007/s10910-010-9677-3

[8] W. Gao, W. Wang, D. Dimitrov, and Y. Wang, "Nano properties analysis via fourth multiplicative $\mathrm{ABC}$ indicator calculating", Arabian journal of chemistry, vol. 11, no. 6, pp. 793-801, Sep. 2018, doi: 10.1016/j.arabjc.2017.12.024

[9] W. Gao, W. Wang, and M. R. Farahani, "Topological indices study of molecular structure in anticancer drugs", Journal of chemistry, vol. 2016, Art ID. 3216327, 2016, doi: 10.1155/2016/3216327

[10] M. Ghorbani and N. Azimi, "Notes on mulyiple Zagreb indices", Iranian journal of mathematical chemistry, vol. 3, no. 2, pp. 137-143, 2012, doi: 10.22052/IJMC.2012.5233 
[11] C. K. Gupta, V. Lokesha, S. B. Shwetha, and P. S. Ranjini, "On the symmetric division deg index of graph", Southeast asian bulletin of mathematics, vol. 40, no. 1, pp. 59-80, 2016. [On line]. Available: https://bit.ly/2Ck4tKF

[12] I. Gutman and K. C. Das, "The first Zagreb indices 30 years after", MATCH communications in mathematical and in computer chemistry, vol. 50, pp. 83-92, 2004. [On line]. Available: https://bit.ly/3ea6XJF

[13] G. Liu, Z. Jia, and W. Gao, "Ontology similarity computing based on stochastic primal dual coordinate technique", Open journal of mathematical sciences, vol. 2, no. 1, pp. 221-227, Aug. 2018, doi: $10.30538 /$ oms 2018.0030

[14] J.-B. Liu and X.-F. Pan, "Minimizing Kirchhoff index among graphs with a given vertex bipartiteness", Applied mathematics and computation, vol. 291, pp. 84-88, Dec. 2016, doi: 10.1016/j.amc.2016.06.017

[15] J.-B. Liu, X.-F. Pan, F.-T. Hu, and F.-F. Hu, "Asymptotic Laplacianenergy-like invariant of lattices", Applied mathematics and computation, vol. 253, pp. 205-214, Feb. 2015, doi: 10.1016/j.amc.2014.12.035

[16] J.-B. Liu, C. Wang, S. Wang, and B. Wei, "Zagreb indices and multiplicative Zagreb indices of Eulerian graphs", Bulletin of the Malaysian Mathematical Sciences Society, vol. 42, no. 1, pp. 67-78, Feb. 2017, doi: $10.1007 / \mathrm{s} 40840-017-0463-2$

[17] J.-B. Liu, J. Zhao, and Z. Zhu, "On the number of spanning trees and normalized Laplacian of linear octagonal-quadrilateral networks", International journal of quantum chemistry, vol. 119, no. 17, pp. Art ID. e25971, 2019, doi: 10.1002/qua.25971

[18] A. Miličević, S. Nikolić, and N. Trinajstić, "On reformulated Zagreb indices," Molecular diversity, vol. 8, no. 4, pp. 393-399, Dec. 2004, doi: 10.1023/B:MODI.0000047504.14261.2a

[19] M. Munir, W. Nazeer, S. Rafique, and S. Kang, "M-Polynomial and related topological indices of nanostar dendrimers", Symmetry, vol. 8, no. 9, Art ID. 97, Sep. 2016, doi: 10.3390/sym8090097 
[20] M. Munir, W. Nazeer, S. Rafique, and S. Kang, "M-Polynomial and degree-based topological indices of polyhex nanotubes", Symmetry, vol. 8, no. 12, Art ID. 149, Dec. 2016, doi: 10.3390/sym8120149

[21] M. Munir, W. Nazeer, S. Rafique, A. R. Nizami, and S. M. Kang, "Some computational aspects of triangular boron nanotube", Symmetry, vol. 9, no. 1, Art ID. 6, Jan. 2016, doi: 10.3390/sym9010006

[22] M. Munir,W. Nazeer,Z. Shahzadi, and S. Kang, "Some invariants of circulantgraphs", Symmetry, vol. 8, no. 11, pp. Art ID. 134, Nov. 2016, doi: 10.3390/sym8110134

[23] S. Noreen and A. Mahmood, "Zagreb polynomials and redefined Zagreb indices for the line graph of carbon nanocones", Open journal of mathematical analysis, vol. 2, no. 1, pp. 66-73, Jun. 2018, doi: 10.30538/psrp-oma2018.0012

[24] M. Randic,, "Characterization of molecular branching", Journal of the American Chemical Society, vol. 97, no. 23, pp.6609-6615, Nov. 1975, doi: 10.1021/ja00856a001

[25] H. M. U. Rehman, R. Sardar, and A. Raza, "Computing topological indices of Hex Board and its line graph", Open journal of mathematical sciences, vol. 1, no. 1, pp. 62-71, 2017, doi: 10.30538/oms2017.0007

[26] M. Riaz, W. Gao, and A. Q. Baig, "M-Polynomials and degree-based topological indices of some families of convex polytopes", Open journal of mathematical sciences, vol. 2, no. 1, pp. 18-28, Feb. 2018, doi: 10.30538/oms2018.0014

[27] M. S. Sardar, S. Zafar, and M. R. Farahani, "The generalized Zagreb index of Capra-designed planar benzenoid series $\mathrm{Ca}_{\mathrm{k}}\left(\mathrm{C}_{6}\right)$ ", Open journal of mathematical sciences, vol. 1, no. 1, pp. 44-51, 2017, doi: 10.30538/oms2017.0005

[28] H. Siddiqui and M. R. Farahani, "Forgotten polynomial and forgotten index of certain interconnection networks", Open journal of mathematical analysis, vol. 1, no. 1, pp. 44-59, Dec. 2017, doi: 10.30538/psrp-oma2017.0005

[29] H. Wiener, "Structural determination of paraffin boiling points", Journal of the American Chemical Society, vol. 69, no. 1, pp. 17-20, Jan. 1947, doi: 10.1021/ja01193a005

[30] L. Yan, M. R. Farahani, and W. Gao, "Distance-based Indices computation of symmetry molecular structures", Open journal of mathematical sciences, vol. 2, no. 1, pp. 323-337, Nov. 2018, doi: 10.30538/oms2018.0038 